\title{
STABILIZATION OF SYSTEMS WITH SECTOR BOUNDED NONLINEARITY BY A SAWTOOTH SAMPLED-DATA FEEDBACK
}

\author{
Alexander N. Churilov \\ Faculty of Mathematics and Mechanics, \\ St. Petersburg State University \\ and \\ Laboratory of Multi-Agent, Distributed and \\ Networked Control Systems, \\ ITMO University, \\ St. Petersburg, Russia \\ a_churilov@mail.ru
}

Article history:

Received 23.10.2019, Accepted 16.12.2019

\begin{abstract}
The paper considers a nonlinear Lur'e type system with a sector bounded nonlinearity. The zero equilibrium of the system may be unstable, so it is stabilized by a periodically sampled feedback signal. Such stabilization problems were previously explored by a number of researches with the help of the zero-order hold $(\mathrm{ZOH})$ control that is kept constant between successive sampling times. The main disadvantage of this method is that the time delay introduced by $\mathrm{ZOH}$ has a destabilizing impact on the closed feedback system, especially in the case when the sampling frequency is sufficiently low and the feedback gain is high. To reduce this effect it is proposed to modify the form of the stabilizing signal. In this paper the reverse sawtooth control is introduced instead of $\mathrm{ZOH}$. The stability criterion is obtained in the form of a feasibility problem for some linear matrix inequalities (LMI). A numerical example demonstrates how the new stabilization method allows to reduce the sampling frequency required for stabilization.
\end{abstract}

\section{Key words}

Nonlinear system, sampled-data stabilization, linear matrix inequalities, integral quadratic constraints

\section{Introduction}

Stability problems for a continuous-time system under a sampled-data feedback attracted much attention in the last decade. This study was largely motivated by appli- cations to networked control systems (see, e. g., [Hespanha et al., 2007]). Sampled-data stabilization of a sector bounded nonlinear system was treated in [Seifullaev and Fradkov, 2015a; Seifullaev and Fradkov, 2015b; Seifullaev and Fradkov, 2015c; Seifullaev and Fradkov, 2016; Zhang et al., 2017; Bryntseva and Fradkov, 2018].

For networked control systems it is desired that the sampling rate of a stabilizing feedback signal should be sufficiently low. As for the intersample control, most of researchers employ the zero-order hold $(\mathrm{ZOH})$ scheme, when the control function is kept constant throughout the sampling interval. It is well known that a system with a $\mathrm{ZOH}$ feedback can be rewritten as a system with a linearly increasing time-varying delay (see, e. g., [Fridman, 2010]), and this delay is the greater, the greater the length of the sampling interval. Such long delays have a destabilizing effect on the system's behavior. It looks reasonable to replace $\mathrm{ZOH}$ for something more sophisticated, which allows to reduce the negative influence of the delay. A reverse sawtooth picewise-linear control was introduced to this end.

This paper continues a series of works [Churilov, 2018; Churilov, 2019a; Churilov, 2019b] devoted to an application of the absolute stability theory to sampled-data stabilization. For the stability analysis of the obtained hybrid system we use the Gelig's averaging method [Gelig, 1982; Gelig and Churilov, 1993b; Gelig and Churilov, 1998] reformulated in terms of linear matrix inequalities [Boyd et al., 1994]. Besides the averaging, we employ such methods as $S$-procedure for multiple quadratic forms [Fradkov and Yakubovich, 1979; Yakubovich, 1992] and integral quadratic constraints (see, e. g., 
[Yakubovich, 1968; Yakubovich, 1988; Megretski and Rantzer, 1997; Yakubovich, 2002]).

The paper is organized as follows. First the system equations are given. Then we present quadratic and integral quadratic constraints that will be needed to prove the main theorem. Further, the main result of the paper is formulated and proven. Finally, we provide an illustrative numerical example. The upper bounds of the sampling period obtained by the theorem of this paper are compared with the similar bounds for $\mathrm{ZOH}$ stabilization.

\section{Problem Setting}

Consider a nonlinear system under a sampled feedback

$$
\begin{aligned}
\dot{x} & =A x(t)+B_{0} f_{0}(t)+B u(t), \\
\sigma_{0}(t) & =C_{0} x(t), \quad \sigma(t)=K x(t) .
\end{aligned}
$$

Here $A, B, B_{0}, K, C_{0}$ are constant matrix coefficients of sizes $p \times p, p \times 1, p \times 1,1 \times p$, and $1 \times p$, respectively, $K$ is a vector of feedback gains.

The function $f_{0}(t)$ is defined as $f_{0}(t)=\varphi\left(\sigma_{0}(t), t\right)$, where the nonlinearity $\varphi(\cdot, \cdot)$ satisfies a sectoral constraint

$$
\mu_{1} \leqslant \frac{\varphi\left(\sigma_{0}, t\right)}{\sigma_{0}} \leqslant \mu_{2} \text { for all } \sigma_{0} \neq 0, t,
$$

$\mu_{1}, \mu_{2}$ are some given numbers (see Figure 1(a)). We also assume that there exists a number $\mu_{0}$ such that the function $\varphi\left(\sigma_{0}, t\right)-\mu_{0} \sigma_{0}$ is bounded for all $\sigma_{0}, t$.

Consider a system with a uniform sampling with the period $T$. The feedback control function $u(\cdot)$ is defined as

$$
u(t+n T)=\sigma(n T)\left(1-\frac{t}{T}\right), \quad 0 \leqslant t<T,
$$

$n=0,1, \ldots$. The function $u(t)$ is a reverse sawtooth wave that linearly ramps downwards (for $\sigma(n T)>0$ ) or upwards (for $\sigma(n T)<0$ ) until it reaches zero (see Figure 1(b)). We are interested in obtaining effectively verifiable conditions for asymptotic to zero of the solutions of system (1)-(4).

\section{The Idea of Averaging}

The pulse averaging technique that we use here originates from the principle of equivalent areas (PEA) [Andeen, 1960a; Andeen, 1960b]. For sector bounded constraints it was firstly obtained in [Gelig, 1982] and the refined in [Gelig and Churilov, 1993a; Gelig and Churilov, 1998]. Following the style of that time, the mathematical statements in [Gelig and Churilov, 1998] were formulated in terms of frequency-domain inequalities, however they can be techically reformulated as LMI by using the celebrated Kalman-Yakubovich-Popov (KYP) lemma (see, e. g., [Popov, 1973; Boyd et al., 1994; Yakubovich et al., 2004]).

Following [Gelig, 1982], compute the average of the $n$th control pulse

$$
v_{n}=\frac{1}{T} \int_{n T}^{n T+T} u(t) d t .
$$

Let the function $w(t)$ be the integrated error of replacing $u(t)$ for its averages:

$$
\begin{aligned}
\dot{w}(t) & =u(t)-v_{n}, \quad n T \leqslant t<n T+T, \\
w(n T) & =0
\end{aligned}
$$

for any $n \geqslant 0$. From (5) it follows that the function $w(t)$ is continuous and turns to zero at all the sampling instants $n T$. The main mathematical technique used in [Gelig and Churilov, 1993a; Gelig and Churilov, 1998] was the construction of quadratic Lyapunov fuctions that depend on $x(t)$ and $w(t)$. The functions $x(t), w(t)$ and the sequence $v_{n}$ were linked by quadratic and integral quadratic constraints. When the sampling frequency tends to infinity, the Gelig's criteria reduce to classical criteria of absolute stability, such as circle or Popov.

The physical aspects of PEA were discussed in [Churilov, 2019a].

Notice that some distant similarity in technique can be found in later works [Briat and Seuret, 2012; Seuret, 2012; Briat, 2013], where loop functionals were introduced and explored. These functionals also become zero at sampling times. However, looped functionals were not related to averaging and were usually added to Lyapunov-Krasovskii functionals.

\section{Quadratic and Integral Quadratic Constraints}

For brevity, denote $t_{n}=n T, n=0,1, \ldots$ Besides the sectoral bound (3), we will need a number of auxiliary constraints. Following the averaging scheme described in the previous section, from (4), (5) we get

$$
v_{n}=\frac{1}{2} \sigma\left(t_{n}\right) .
$$

Define a piecewise constant function

$$
v(t)=v_{n}, \quad t_{n} \leqslant t<t_{n+1} .
$$

By a straightforward calculation, from (6) we have

$$
w(t)=\frac{v_{n}}{T}\left(t_{n+1}-t\right)\left(t-t_{n}\right), \quad t_{n} \leqslant t \leqslant t_{n+1} .
$$

Notice that $|w(t)| \leqslant \frac{1}{4} T|v(t)|$. Additionally, a quadratic constraint

$$
v(t) w(t) \geqslant 0, \quad t_{n}<t<t_{n+1},
$$

is valid. By a direct calculation we get

$$
\begin{aligned}
& \int_{t_{n}}^{t_{n+1}} w(t) d t=\frac{1}{6} v_{n} T^{2} \\
& \int_{t_{n}}^{t_{n+1}} w(t)^{2} d t=\frac{1}{30} v_{n}^{2} T^{3} .
\end{aligned}
$$



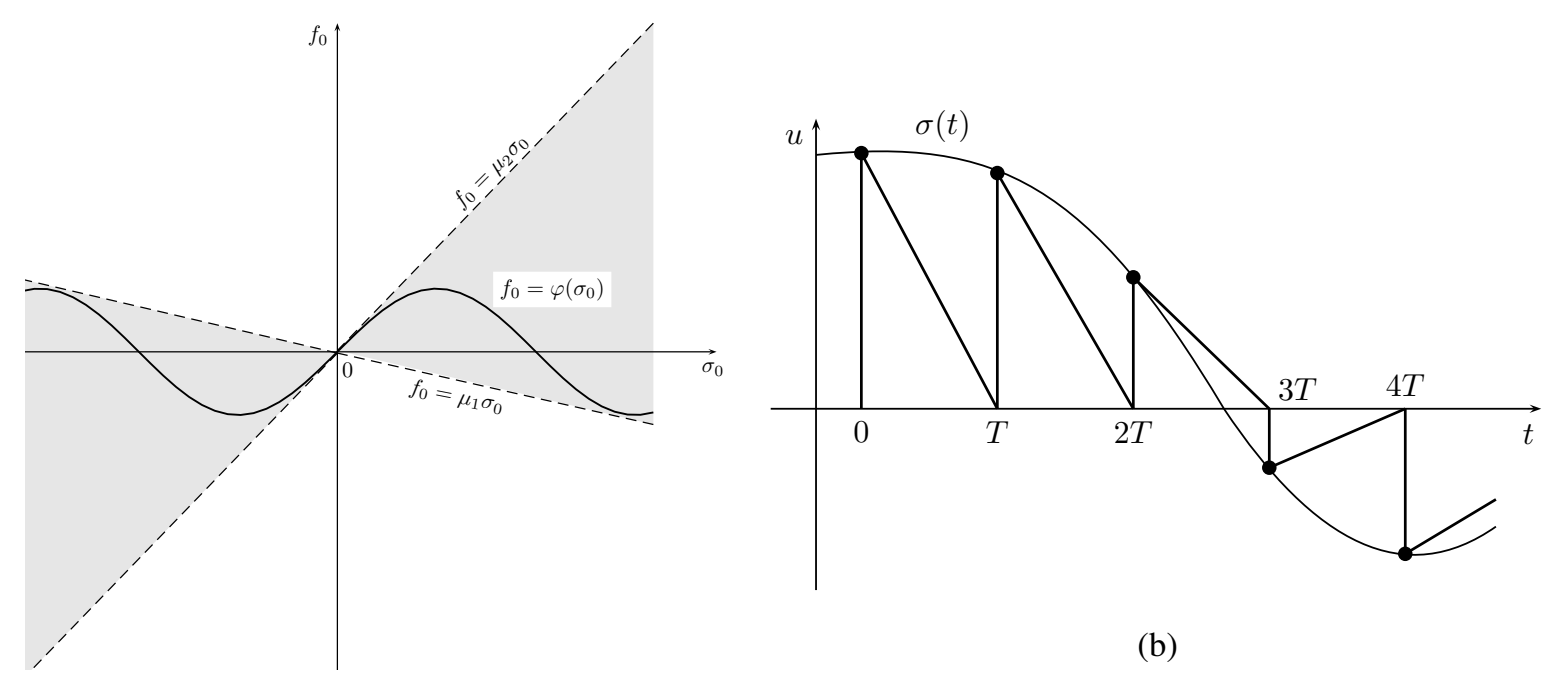

(b)

(a)

Figure 1. (a) An example of a bound sector for $\varphi\left(\sigma_{0}, t\right) \equiv \varphi\left(\sigma_{0}\right)$. (b) The sampling scheme.

Equalities (8) and (11) imply

$$
T \int_{t_{n}}^{t_{n+1}} v(t) w(t) d t=5 \int_{t_{n}}^{t_{n+1}} w(t)^{2} d t .
$$

Equation (12) can be rewritten as an integral quadratic constraint

$$
\int_{t_{n}}^{t_{n+1}}\left(T v(t) w(t)-5 w(t)^{2}\right) d t=0
$$

for all $n \geqslant 0$.

Introduce a picewise-continuous function

$$
\xi(t)=\sigma(t)-\sigma\left(t_{n}\right)-K B w(t)
$$

for $t_{n} \leqslant t<t_{n+1}, n \geqslant 0$. Hence

$$
\xi(t)=K x(t)-2 v(t)-K B w(t), \quad t \geqslant t_{0} .
$$

Obviously, $\xi\left(t_{n}^{+}\right)=0, n \geqslant 0$ and

$$
\dot{\xi}(t)=K A x(t)+K B_{0} f_{0}(t)+K B v(t)
$$

for $t \neq t_{n}$. Then Wirtinger inequality [Gelig and Churilov, 1993b; Gelig and Churilov, 1998] implies

$$
\int_{t_{n}}^{t_{n+1}} \xi(t)^{2} d t \leqslant \frac{4 T^{2}}{\pi^{2}} \int_{t_{n}}^{t_{n+1}} \dot{\xi}(t)^{2} d t
$$

for $n \geqslant 0$. Additionally, we have an obvious constraint

$$
\int_{t_{n}}^{t_{n+1}} \xi(t) \dot{\xi}(t) d t \geqslant 0
$$

If we introduce a vector column

$$
X(t)=\operatorname{col}\left\{x(t), f_{0}(t), v(t), w(t)\right\},
$$

then from (14), (15) we obtain

$$
\xi(t)=D X(t), \quad \dot{\xi}(t)=D_{1} X(t),
$$

where

$$
\begin{aligned}
D & =\left[\begin{array}{cccc}
K & 0 & -2 & -K B
\end{array}\right] \\
D_{1} & =\left[\begin{array}{ccccc}
K A & K B_{0} & K B & 0
\end{array}\right] .
\end{aligned}
$$

\section{The Main Statement}

Theorem 1. Assume that there exist a symmetric positive definite $p \times p$ matrix $H$, nonnegative numbers $\varepsilon_{0}$, $\varepsilon_{2}, \varepsilon_{3}$ and a number $\varepsilon_{1}$ of any sign, such that

$$
\begin{aligned}
\Pi & +\varepsilon_{2}\left(\Delta^{2} D_{1}^{\top} D_{1}-D^{\top} D\right) \\
& +\varepsilon_{3}\left(D^{\top} D_{1}+D_{1}^{\top} D\right)<0,
\end{aligned}
$$

where $\Delta=2 T / \pi, \Pi$ is a symmetric $(p+3) \times(p+3)$ matrix with the components

$$
\begin{aligned}
& \Pi_{11}=H A+A^{\top} H-\varepsilon_{0} \mu_{1} \mu_{2} C_{0}^{\top} C_{0}, \\
& \Pi_{12}=H B_{0}+\frac{1}{2} \varepsilon_{0}\left(\mu_{1}+\mu_{2}\right) C_{0}^{\top}, \\
& \Pi_{13}=H B, \quad \Pi_{14}=-A^{\top} H B, \\
& \Pi_{22}=-\varepsilon_{0}, \quad \Pi_{23}=0, \quad \Pi_{24}=-B^{\top} H B_{0}, \\
& \Pi_{33}=0, \quad \Pi_{34}=T \varepsilon_{1}-B^{\top} H B, \\
& \Pi_{44}=-10 \varepsilon_{1}
\end{aligned}
$$

and $D, D_{1}$ are defined by (20). Then any solution of system (1)-(4) satisfies $x(t) \rightarrow 0$ as $t \rightarrow+\infty$ and $x(t) \in L_{2}\left[t_{0},+\infty\right)$. 
Proof. We will apply the $S$-procedure with multiple quadratic forms [Fradkov and Yakubovich, 1979; Yakubovich, 1992]. Consider a $(p+3)$-dimensional vector column

$$
X=\operatorname{col}\left\{x, f_{0}, v, w\right\},
$$

and introduce a quadratic form

$$
\begin{aligned}
\Phi(X)= & \varepsilon_{0}\left(\mu_{2} C_{0} x-f_{0}\right)\left(f_{0}-\mu_{1} C_{0} x\right) \\
& +2 \varepsilon_{1}\left(T v w-5 w^{2}\right) \\
& +\varepsilon_{2}\left(\Delta^{2}\left(D_{1} X\right)^{2}-(D X)^{2}\right) \\
& +2 \varepsilon_{3} D_{1} X D X .
\end{aligned}
$$

(The numbers $\varepsilon_{i}, 0 \leqslant i \leqslant 3$, play the role of Lagrange multipliers.)

Inequality (21) can be rewritten as

$$
\begin{array}{r}
2(x-B u)^{\top} H\left(A x+B_{0} f_{0}+B v\right) \\
+\Phi(X) \leqslant-\delta_{0}\|X\|^{2}
\end{array}
$$

for all vector columns $X$ with coordinates (23). Here $\delta_{0}$ is a sufficiently small positive number. Let us take a Lyapunov function $V(x, w)=(x-B w)^{\top} H(x-B w)$. Then along the solutions of system (1)-(4) inequality (25) implies

$$
\dot{V}(x(t), w(t))+\Phi(X(t)) \leqslant-\delta_{0}\|X(t)\|^{2}
$$

for any sampling interval $t_{n}<t<t_{n+1}$. From quadratic bounds (3), (10) and integral quadratic constraints (13), (16), (17) we obtain

$$
\int_{t_{n}}^{t_{n+1}} \Phi(X(t)) d t \geqslant 0
$$

for all $n \geqslant 0$. Integrating (26) and taking (27) into account, we get

$$
\begin{aligned}
& V\left(x\left(t_{n+1}\right), 0\right)- V\left(x\left(t_{n}\right), 0\right) \\
& \leqslant-\delta_{0} \int_{t_{n}}^{t_{n+1}}\|x(t)\|^{2} d t-\delta_{0} T v_{n}^{2}
\end{aligned}
$$

for all $n \geqslant 0$. The rest of the proof reproduces the proof of Theorem 1 [Churilov, 2018].

\section{Necessary Conditions for the Fulfillment of the Main Statement}

Preposition 1. Let conditions of Theorem 1 be satisfied. Then the matrix

$$
A_{\mu}=A+\mu B_{0} C_{0}+\frac{1}{2} B K
$$

is Hurwitz stable for any number $\mu, \mu_{1} \leqslant \mu \leqslant \mu_{2}$.

Proof. Let us put

$$
f_{0}=\mu C_{0} x, \quad v=\frac{1}{2} K x, \quad w=0
$$

in (24), (25). Then $D X=0$ and (25) implies

$$
H A_{\mu}+A_{\mu}^{\top} H<0 .
$$

Since $H>0$, the Hurwitz stability of $A_{\mu}$ follows.

\section{Numerical Example}

Consider the system (see [Seifullaev and Fradkov, 2015c])

$$
\begin{aligned}
& \dot{x}_{1}=-2 x_{1}+\sin x_{2}, \\
& \dot{x}_{2}=x_{1}-x_{2}+2 \sin x_{2}-u(t) .
\end{aligned}
$$

Here $\sigma_{0}(t)=x_{2}(t), \varphi_{0}\left(\sigma_{0}\right)=\sin \sigma_{0}$, and we can take $\mu_{1}=-0.2173, \mu_{2}=1$. The control function $u(t)$ is defined by (4), where $\sigma(t)=k x_{2}(t)$ and $k$ is a scalar feedback gain. Thus system (29) can be written in the form of (1)-(4) with

$$
\begin{aligned}
A & =\left[\begin{array}{cc}
-2 & 0 \\
1 & -1
\end{array}\right], \quad B_{0}=\left[\begin{array}{l}
1 \\
2
\end{array}\right], \quad B=\left[\begin{array}{c}
0 \\
-1
\end{array}\right], \\
C_{0} & =\left[\begin{array}{ll}
0 & 1
\end{array}\right], \quad K=\left[\begin{array}{ll}
0 & k
\end{array}\right] .
\end{aligned}
$$

Feasibility of inequalities (21) of Theorem 1 was explored with the help of YALMIP software package for interface and SeDuMi solver for semidefinite programming [Löfberg, 2004; Sturm, 1999].

System (29) was previously studied in [Seifullaev and Fradkov, 2015c] with the use of $\mathrm{ZOH}$ control

$$
u(t)=\sigma\left(t_{n}\right), \quad t_{n} \leqslant t<t_{n+1} .
$$

The results of computer experiments are consolidated in Table 1. The computer experiment was produced for the values $k=2,3,4,5,10$ (see column 1 ). The maximal values of $T$ computed with the help of Theorem 1 are given in column 2. Column 3 contains the maximal values of $T$ obtained by the Lyapunov-Krasovskii method for the case of $\mathrm{ZOH}$ control in [Seifullaev and Fradkov, 2015c] (the value $k=4$ was not considered). For $k=2,3$ Theorem 1 gives no feasible values, but for $k=5,10$ the maximum of $T$ obtained from Theorem 1 is greater than that was found in [Seifullaev and Fradkov, 2015c].

The comparison of the reverse sawtooth (RS) control and the $\mathrm{ZOH}$ control is presented in Table 2. The maximal values of $T$ are obtained by a direct computer simulation under the $T$-periodic sampling (i. e., they are irrelevant to any stability criteria). It is seen that for the same feedback gain $k$ the RS control provides a sampling pe$\operatorname{riod} T$ approximately 1.9 times greater than that of $\mathrm{ZOH}$ control.

Some transients for system (29) with $k=5$ and the $T$-periodic sampling are shown in Figures 2,3. With the increase of $T$ the zero equilibrium turns from a stable node to a stable focus. With the further increase of $T$ this focus loses stability. 
Table 1. Maximal values of $T$ computed according to Theorem 1 and their comparison with the values obtained in [Seifullaev and Fradkov, 2015c] for ZOH feedback

\begin{tabular}{c|c|c}
\hline$k$ & $T$ & $T$ \\
& $($ Theorem 1) & (Seifullaev, Fradkov, 2015) \\
\hline \hline 2 & - & 0.68 \\
3 & - & 0.53 \\
4 & 0.56 & $?$ \\
5 & 0.51 & 0.35 \\
10 & 0.29 & 0.187 \\
\hline
\end{tabular}

Table 2. Maximal values of $T$ simulated for Reverse Sawtooth (RS) feedback and for $\mathrm{ZOH}$ feedback

\begin{tabular}{c|c|c}
\hline$k$ & Simulated $T(\mathrm{RS})$ & Simulated $T(\mathrm{ZOH})$ \\
\hline \hline 2 & - & 1.21 \\
3 & 1.38 & 0.71 \\
4 & 0.98 & 0.51 \\
5 & 0.77 & 0.40 \\
10 & 0.38 & 0.20 \\
\hline
\end{tabular}

\section{Acknowledgement}

The work was supported by the Russian Foundation for Basic Research (Grant 17-01-00102-a) and by the Government of Russian Federation (Grant 08-08).

\section{Conclusion}

The paper introduces a new control function based on the reverse sawtooth signal wave, which can significantly increase the sampling period compared with the zero-order hold. Based on the Gelig-Yakubovich mathematical technique, the paper suggests an easily verifiable stability criterion formulated in terms of linear matrix inequalities. Simulation shows its reasonable conservatism and an agreement with the previously obtained results.

\section{References}

Andeen, R. E. (1960a). Analysis of pulse duration sampled-data systems with linear elements. IRE Trans. Autom. Control, 5 (4), pp. 306-313.

Andeen, R. E. (1960b). The principle of equivalent areas. Trans. AIEE (Applications and Industry), (79), pp. 332-336.

Boyd, S., El Ghaoui, L., Feron, E., and Balakrishnan, V. (1994). Linear Matrix Inequalities in System and Control Theory. SIAM, Philadelphia.

Briat, C. (2013). Convex conditions for robust stability analysis and stabilization of linear aperiodic impulsive and sampled-data systems under dwell-time constraints. Automatica, 49 (11), pp. 3449-3457.
Briat, C. and Seuret, A. (2012). A looped-functional approach for robust stability analisys of linear impulsive systems. Syst. Control Lett., 61 (10), pp. 980-988.

Bryntseva, T. A. and Fradkov, F. L. (2018). Frequencydomain estimates of the sampling interval in multirate nonlinear systema by time-delay approach. Int. J. Control.

Churilov, A. N. (2018). On an application of the absolute stability theory to sampled-data stabilization. Math. Probl. Engin., 2018. Article ID 3169609, 9 pages.

Churilov, A. N. (2019a). Stability analysis of Lur'e systems with a pulse-modulated feedback. Cybern. Phys., $8(2)$, pp. 58-68.

Churilov, A. N. (2019b). Stabilization of the Lur'e system by a pulse-width-modulated control. Funct. Diff. Equat., 26 (1-2), pp. 51-59.

Fradkov, A. L. and Yakubovich, V. A. (1979). The $S$ procedure and duality relations in nonconvex problems of quadratic programming. Vestnik Leningrad Univ. Math., 6, pp. 101-109.

Fridman, E. (2010). A refined input delay approach to sampled-data control. Automatica, 46 (2), pp. 421427.

Gelig, A. Kh. (1982). Frequency criterion for nonlinear pulse systems stability. Syst. Control Lett., 1 (6), pp. 409-412.

Gelig, A. Kh. and Churilov, A. N. (1993a). Oscillations and Stability of Nonlinear Impulsive Systems. St. Petersburg State Univ., St. Petersburg. (Russian).

Gelig, A. Kh. and Churilov, A. N. (1993b). Popovtype stability criterion for the functional-differential equations describing pulse-modulated control systems. Funct. Diff. Equat., 1, pp. 95-107.

Gelig, A. Kh. and Churilov, A. N. (1998). Stability and Oscillations of Nonlinear Pulse-modulated Systems. Birkhäuser, Boston.

Hespanha, J. P., Naghshtabrizi, P., and Xu, Y. (2007). A survey of recent results in networked control systems. Proc. IEEE, 95 (1), pp. 138-162.

Löfberg, J. (2004). YALMIP : A toolbox for modeling and optimization in MATLAB. In IEEE Int. Symp. Computer Aided Control Syst. Design (CACSD), Taipei, Taiwan, pp. 284-289.

Megretski, A. and Rantzer, A. (1997). System analysis via integral quadratic constraints. IEEE Trans. Automat. Contr., 42 (6), pp. 819-830.

Popov, V. M. (1973). Hyperstability of Control Systems. Springer, Berlin.

Seifullaev, R. E. and Fradkov, A. L. (2015a). Linear matrix inequality-based analysis of the discretecontinuous nonlinear multivariable systems. Automat. Remote Control, 76 (6), pp. 989-1004.

Seifullaev, R. E. and Fradkov, A. L. (2015b). Robust nonlinear sampled-data system analysis based on Fridman's method and S-procedure. Int. J. Robust Nonlin. Control, 26(2), pp. 201-217. 


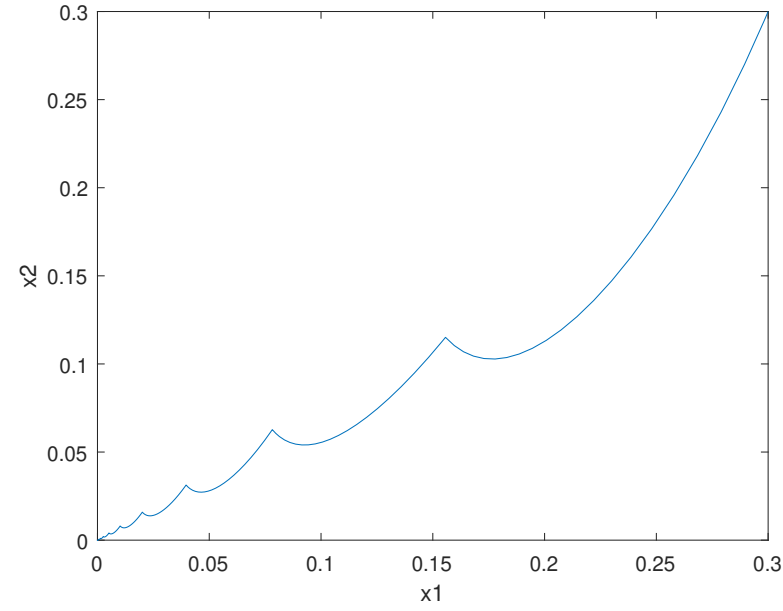

(a)

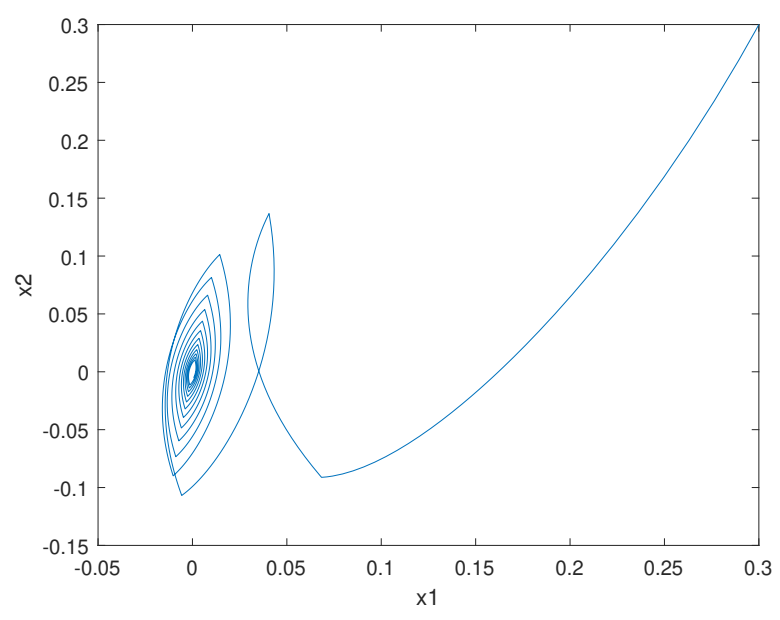

(b)

Figure 2. A trajectory enters zero with the increase of time. (a) Non-oscillative behavior, $k=5, T=0.5$ (b) Oscillative behavior, $k=5, T=0.75$

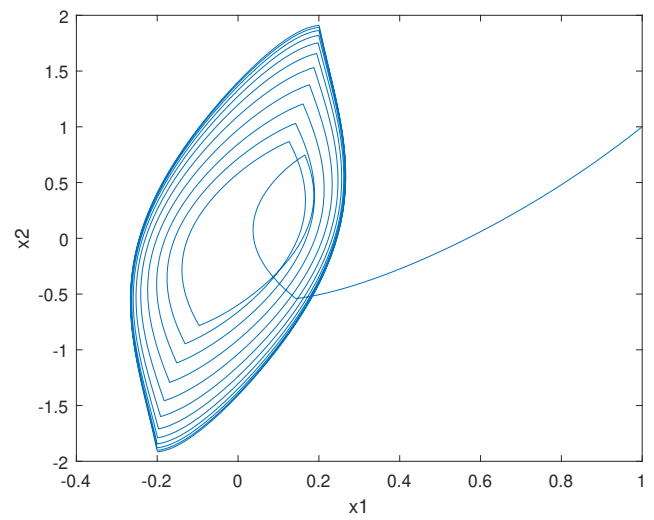

Figure 3. Stabilization fails, the trajectory tends to infinity (oscillative behavior), $k=5, T=0.8$

Seifullaev, R. E. and Fradkov, A. L. (2015c). Sampleddata control of nonlinear systems based on Fridman's analysis and passification design. IFAC-PapersOnLine, 48 (11), pp. 685-690.

Seifullaev, R. E. and Fradkov, A. L. (2016). Eventtriggered control of sampled-data nonlinear systems. IFAC-PapersOnLine, 49 (14), pp. 12-17.

Seuret, A. (2012). A novel stability analisys of linear systems under asynchronous samplings. Automatica,
48 (1), pp. 177-182.

Sturm, J. F. (1999). Using SeDuMi 1.02, a MATLAB toolbox for optimization over symmetric cones. Optimiz. Methods Software, 11-12, pp. 625-653.

Yakubovich, V. A. (1968). On impulsive control systems with a pulse width modulation. Doklady Akad. Nauk SSSR, 180, pp. 283-285. (Russian).

Yakubovich, V. A. (1988). Dichotomy and absolute stability of nonlinear systems with periodically nonstationary linear part. Syst. Contr. Lett., 11 (3), pp. 211218.

Yakubovich, V. A. (1992). Nonconvex optimization problem: The infinite-horizon linear-quadratic control problem with quadratic constraints. Syst. Contr. Lett., 19(1), pp. 13-22.

Yakubovich, V. A. (2002). Popov's method and its subsequent development. Europ. J. Contr., 2 (3), pp. 200208.

Yakubovich, V. A., Leonov, G. A., and Gelig, A. Kh. (2004). Stability of Stationary Sets in Control Systems with Discontinuous Nonlinearities. World Scientific, Singapore.

Zhang, F., Mazo Jr., M., and van de Wouw, N. (2017). Absolute stabilization of Luré systems under eventtriggered feedback. IFAC PapersOnLine, 50(1), pp. 15301-15306. 